\title{
MULTIFUNCTIONAL MIMO SYSTEMS: A COMBINED DIVERSITY AND MuLTIPLEXINg Design PERSPECTIVE
}

\author{
MOHAMmEd El-HAJJAR AND LAJOS HANZO, UNIVERSITY OF SOUTHAMPTON
}

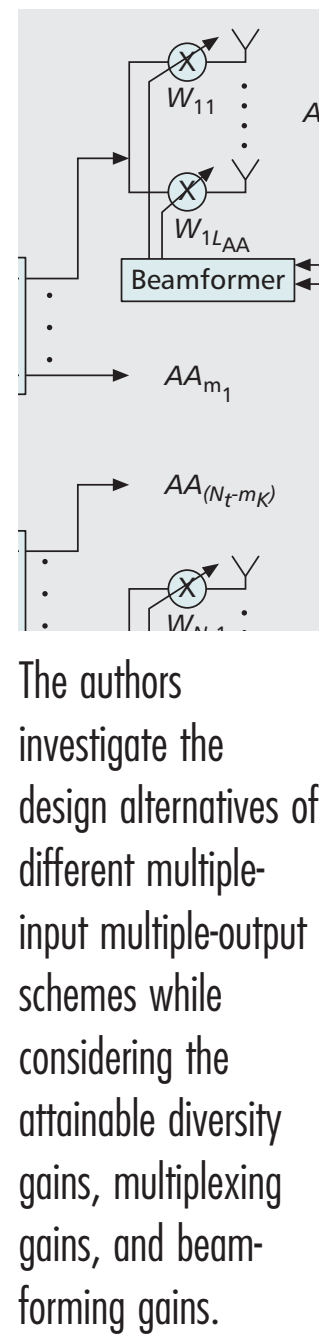

Recently, there has been an urgent demand for flexible and bandwidth-efficient transceivers capable of supporting the explosive expansion of the Internet and the continued dramatic increase

The financial support of Vodafone under the auspices of the Dorothy Hodgkin postgraduate award and that of the European Community under Seventh Framework Programme grant agreement ICT OPTIMIX nINFSO-ICT214625 is gratefully acknowledged. in demand for high-speed multimedia wireless services. Advances in channel coding made it feasible to approach Shannon's capacity limit in systems equipped with a single antenna [1], but fortunately these capacity limits can be further extended with the aid of multiple antennas. Recently, multiple-input multiple-output (MIMO) systems have attracted considerable research attention and it is considered as one of the most significant technical breakthroughs in contemporary communications.

Explicitly, MIMO schemes can be categorized as diversity techniques, multiplexing schemes, multiple access methods, and beamforming as well as multifunctional MIMO arrangements, as shown in Fig. 1. Diversity is the technique where the receiver receives several replicas of the same transmitted signal, while assuming that at least some of them are not severely attenuated. Spatial diversity can be attained by employing multiple antennas at the transmitter or the receiver. Multiple antennas can be used to transmit and receive appropriately encoded replicas of the same information sequence in order to achieve diversity and hence obtain an improved bit error rate (BER) performance. On the other hand, multiplexing techniques were designed in order to improve the attainable spectral efficiency of the system by transmitting the signals independent of each of the transmit antennas. In the context of diversity and multiplexing techniques, the antennas are spaced as far apart as possible so that the signals transmitted to or received by the different antennas experience independent fading, and hence we attain the highest possible diversity or multiplexing gain. Additionally, multiple antennas can also be used in order to improve the signal-to-noise ratio (SNR) at the receiver or the signal-to-interference-plus-noise ratio (SINR) in a multi-user scenario. This can be achieved by employing beamforming techniques, where the antenna gain is increased in the direction of the desired user, whilst reducing the gain towards the interfering users.

A simple spatial diversity technique, which does not involve any loss of bandwidth, is constituted by the employmexnt of multiple antennas 
at the receiver, where several techniques can be employed for combining the independently fading signal replicas. In case of narrowband frequency-flat fading, the optimum combining strategy in terms of maximizing the SNR at the combiner output is maximum ratio combining (MRC). Additionally, other combining techniques have been proposed in the literature, as shown in Fig. 1, including equal gain combining (EGC) and selection combining (SC). All three combining techniques are said to achieve full diversity order, which is equal to the number of receive antennas.

On the other hand, several transmit - rather than receive - diversity techniques have also been proposed in the literature [2-5], as shown in Fig. 1. In [2] Alamouti proposed a witty transmit diversity technique using two transmit antennas, the key advantage of which was the employment of low-complexity single-receiveantenna-based detection, which avoids the more complex joint detection of multiple symbols. The decoding algorithm proposed in [2] can be generalized to an arbitrary number of receive antennas using MRC, EGC, or SC. Alamouti's achievement inspired Tarokh et al. [3] to gener-

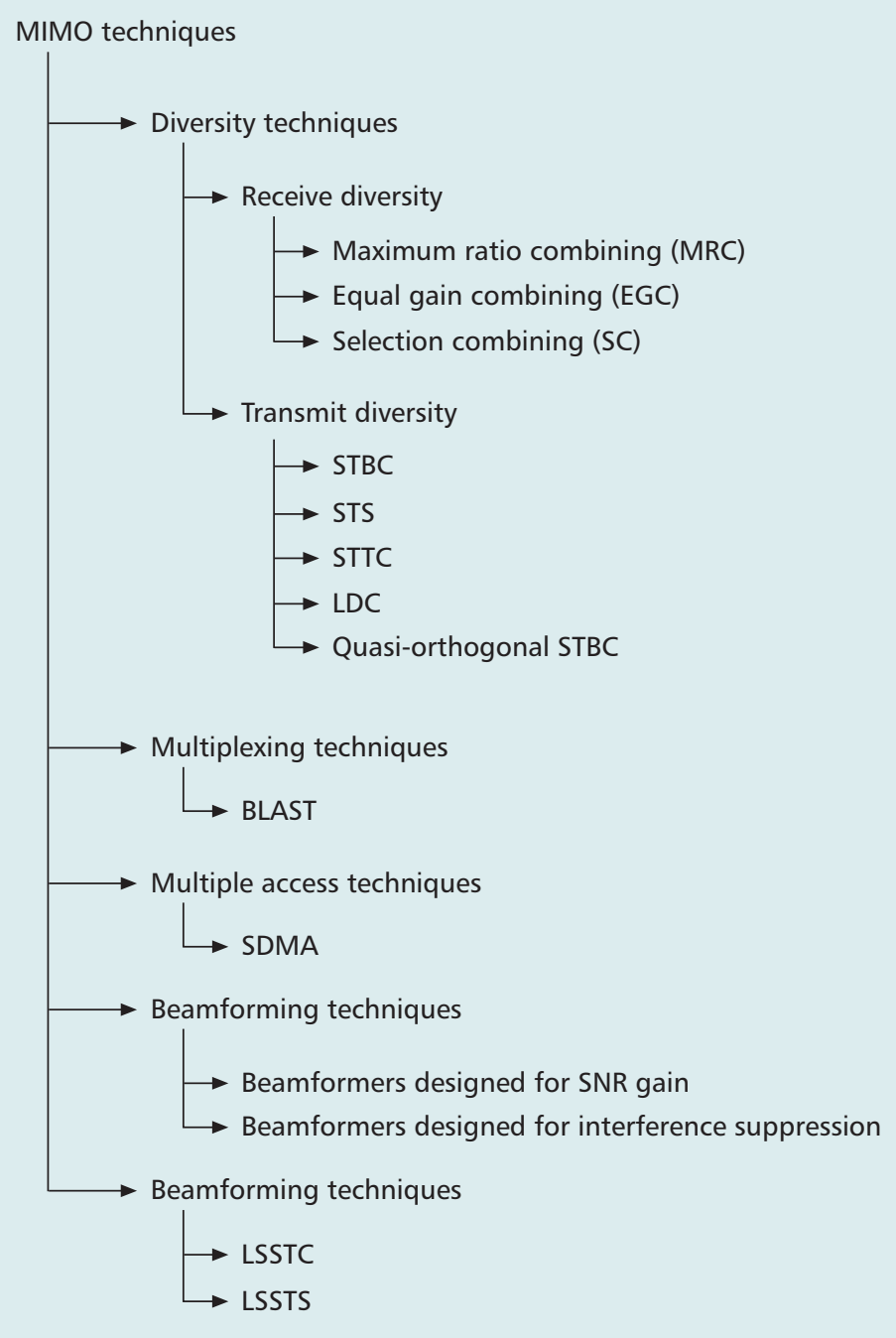

Figure 1. Classification of MIMO techniques. alize the concept of transmit diversity schemes to more than two transmit antennas, contriving the generalized concept of orthogonal spacetime block codes (OSTBCs). The family of OST$\mathrm{BCs}$ is capable of attaining the same diversity gain as space-time trellis codes (STTC) [4] at lower decoding complexity when employing the same number of transmit antennas. However, a disadvantage of OSTBCs when compared to STTCs is that they employ unsophisticated repetition coding and hence provide no coding gain. Furthermore, inspired by the philosophy of STBCs, Hochwald et al. [6] proposed the transmit diversity concept known as space-time spreading (STS) for the downlink of wideband code-division multiple access (WCDMA) that is capable of achieving the highest possible transmit diversity gain.

Regretfully, the OSTBC and STS designs of $[3,6]$ contrived for more than two transmit antennas result in a reduction of the achievable throughput per channel use when complex-valued constellations are used. An alternative idea invoked for constructing full-rate STBCs for complex-valued modulation schemes and more than two antennas was suggested in [7]. Here the strict constraint of perfect orthogonality was relaxed in favor of achieving a higher data rate. The resultant STBCs were referred to as quasiorthogonal STBCs [7].

The OSTBC and STS designs offer - at best - the same data rate as an uncoded singleantenna system, but they provide improved BER performance compared to the family of singleantenna-aided systems, since they attain a diversity gain. In contrast to this, several high-rate space-time transmission schemes having a normalized rate higher than unity have been proposed in the literature. For example, high-rate space-time codes that are linear in both space and time, the family of so-called linear dispersion codes (LDCs), was proposed in [5]. LDCs strike a flexible trade-off between emulating space-time coding and/or spatial multiplexing. The attractive concept of LDCs invokes a matrixbased linear modulation framework, where each space-time transmission matrix is generated by a linear combination of so-called dispersion matrices, and the weights of the components are determined by the transmitted symbol vector.

OSTBCs and STTCs are capable of providing an attractive diversity gain for the sake of improving the achievable system performance. However, this BER performance improvement is often achieved at the expense of a rate loss, since OSTBCs and STTCs may result in a throughput loss compared to single-antennaaided systems. As a design alternative, a specific class of MIMO systems was designed for improving the attainable spectral efficiency of the system by transmitting different parallel signal streams independently over each of the transmit antennas, hence resulting in a multiplexing gain. This class of MIMOs subsumes Bell Labs' layered dpace-yime (BLAST) scheme and its relatives [8]. The BLAST scheme aims to increasie the system throughput in terms of the number of bits per symbol that can be transmitted in a given bandwidth at a given integrity.

In contrast to the family of BLAST schemes, 
where multiple antennas are activated by a single user for increasing the user's throughput, space-division multiple access (SDMA) [9] employs multiple antennas for the sake of supporting multiple users. SDMA exploits the unique user-specific channel impulse responses (CIRs) of different users to separate their received signals.

On the other hand, in beamforming arrangements [9] typically $\lambda / 2$-spaced antenna elements are used for the sake of creating a spatially selective transmitter/receiver beam, where $\lambda$ represents the carrier's wavelength. Beamforming is employed for providing angular receiver selectivity by mitigating the effects of various interfering signals, provided that they arrive from sufficiently different angular directions. Additionally, beamforming is capable of suppressing the effects of co-channel interference, hence allowing the system to support multiple users by angularly separating them. Again, this angular separation becomes feasible only on condition that the corresponding users are separable in terms of the angle of arrival of their beams.

Finally, multifunctional MIMOs, as the term suggests, combine the benefits of several MIMO schemes including diversity gains, multiplexing gains, and beamforming gains. As mentioned earlier, V-BLAST is capable of achieving the maximum attainable multiplexing gain, while STBC may attain the maximum achievable antenna diversity gain facilitated by the number of independently fading diversity channels. Hence, it was proposed in [10] to combine these two techniques in order to provide both antenna diversity and spectral efficiency gains. On the other hand, in [11] the authors presented a transmission scheme referred to as double spacetime transmit diversity (D-STTD), which consists of two STBC layers at a transmitter equipped with four transmit antennas, while the receiver is equipped with two antennas. Furthermore, in order to achieve additional performance gains, beamforming has been combined with both spatial diversity as well as spatial multiplexing techniques. STBC has been combined with beamforming in order to attain an improved SNR gain in addition to the diversity gain [12, 13].

This contribution provides a lighthearted perspective on further research advances in the field of multifunctional MIMO systems, and demonstrates how diversity, multiplexing, and beamforming gains are achieved by multifunctional MIMOs. More explicitly, in the next section we elaborate on the design of multifunctional MIMO schemes and describe the evolution of the idea of multifunctional MIMO systems. We quantify the achievable performance of the different MIMO schemes. A comparison of the different MIMO schemes expressed in terms of their diversity, multiplexing, and beamforming gains is then presented, followed by our conclusions in the final section.

\section{Multifunctional MIMO SyStems}

Space-time codes have been designed for the sake of attaining the highest possible diversity gain, while the V-BLAST scheme was designed

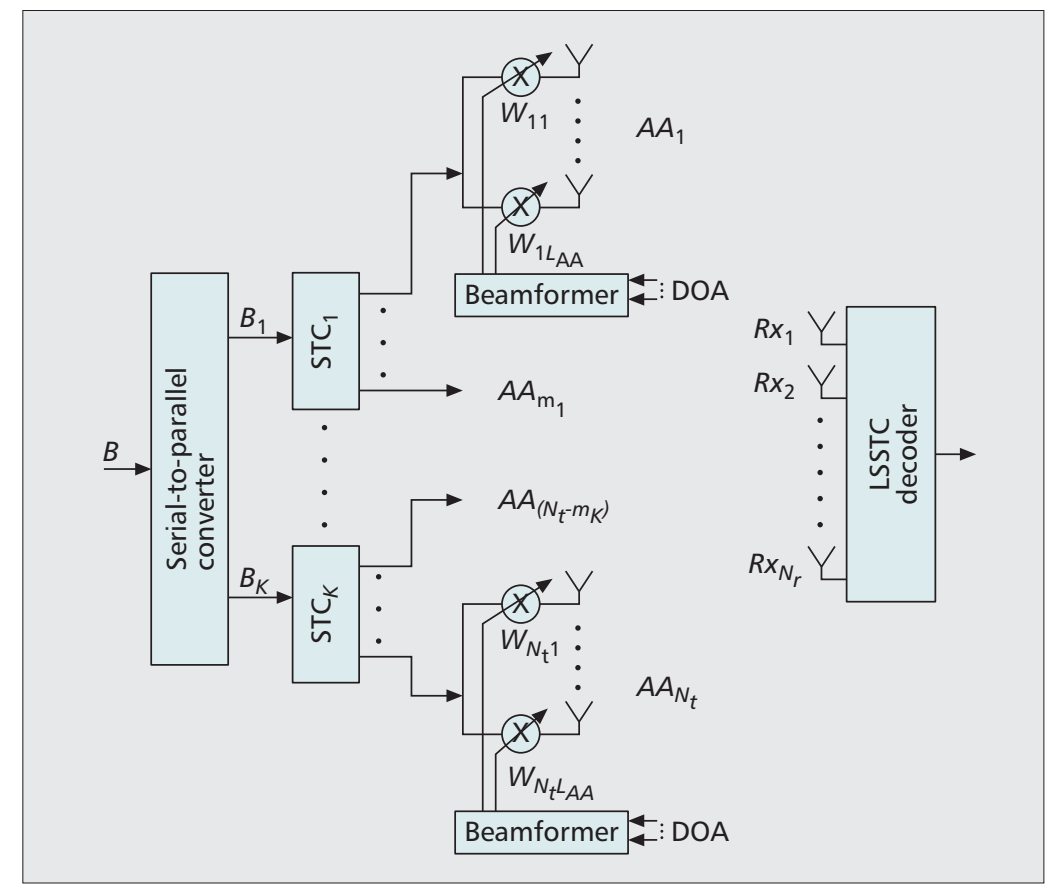

Figure 2. Multi-functional MIMO system block diagram.

for attaining the maximum achievable multiplexing gain equal to the number of transmit antennas. Additionally, beamforming schemes have been designed in order to attain an SNR gain. Therefore, the appealing concept of multifunctional MIMO schemes designed for combining the benefits of STBC, BLAST, and beamforming schemes arises in order to provide diversity, multiplexing, and SNR gains.

Figure 2 shows the block diagram of a general multifunctional MIMO scheme that can combine the benefits of space-time coding (STC), BLAST, and beamforming. The system's architecture seen in the figure has $N_{t}$ transmit antenna arrays (AAs) spaced sufficiently far apart in order to experience independent fading, and hence achieve transmit diversity and/or multiplexing. The $L_{A A}$ number of elements of each $\mathrm{AA}$ is spaced at a distance of $\lambda / 2$ for the sake of achieving a beamforming gain. Furthermore, the receiver is equipped with $N_{r}$ antennas. According to Fig. 2, a block of $B$ input information symbols is serial-to-parallel converted to $K$ groups of symbol streams of length $B_{1}, B_{2}, \cdots, B_{K}$, where $B_{1}+B_{2}+\cdots+B_{K}=B$. Each group of $B_{k}$ symbols, $k \in[1, K]$, is then encoded by a component space-time code $\mathrm{STC}_{k}$ associated with $m_{k}$ transmit AAs, where $m_{1}+m_{2}+\cdots+m_{K}=N_{t}$.

The STC employed can be OSTBC, STTC, or STS for the sake of attaining a diversity gain. The data transmitted from each component STC is independent of the data transmitted from all the other STCs, which results in a multiplexing gain, where the throughput of the multifunctional MIMO scheme is $K$ times that of a scheme employing a single STC. The multiplexing gain is attained by considering each STC as a layer in a BLAST scheme. Furthermore, the data is transmitted using antenna arrays that can be used for attaining a beamforming gain.

In [10] a dual-functional MIMO scheme was 
One configuration

can employ two four-

antenna-aided STCs,

while another

configuration may

employ four twin-

antenna-aided STCs.

The favored

configuration will

depend on the

specific application

as well as on the

required performance

and throughput. proposed that combines the benefits of $\mathrm{V}$ BLAST and OSTBC. The scheme presented in [10] considered transmissions over OSTBCs where several parallel OSTBC blocks were capable of transmitting independent data. Hence, the scheme of [10] was capable of attaining the diversity gain of the OSTBC as well as the multiplexing gain due to using several independent OSTBC layers. However, a drawback of the scheme in [10] was that the decoder implemented group successive interference cancellation (GSIC) that did not take into account the antenna-specific received signal power at the different OSTBC layers for the sake of ordering the layers before interference cancellation.

On the other hand, dual-functional MIMO schemes that combine STBC with beamforming were proposed in $[12,13]$. These schemes benefit from the diversity gain of the STBCs and the SNR gain of the beamformer. In [12] the authors combined conventional transmit beamforming with OSTBC, assuming that the transmitter has partial knowledge of the channel, and derived a performance criterion for improving the system performance. Furthermore, the scheme presented in [13] combined the twin-antenna-aided Alamouti STBC with ideal beamforming, where it was assumed that the transmitter has full knowledge of the channel as well as the signal's direction of arrival at the receiver in order to show that the system can attain better performance while attaining the maximum achievable diversity order at a unity rate.

Inspired by the performance improvements reported in [10, 12, 13], El-Hajjar et al. proposed in [14] a trifunctional MIMO scheme that combines diversity gain with multiplexing gain and beamforming gain. The MIMO scheme of [14] was referred to as a layered steered space-time code (LSSTC), where the parallel data streams were encoded by OSTBC layers, and each layer might have a different OSTBC structure. Additionally, the decoder of the LSSTC scheme employed an ordering strategy for decoding the different layers in order to improve the achievable performance.

The decoder of the LSSTC scheme may apply GSIC based on the classic Zero Forcing (ZF) algorithm [10] for decoding the received signal. The most beneficial decoding order of the STC layers is determined on the basis of detecting the highest-power layer first for the sake of high correct detection probability. For simplicity, let us consider the case of $K=2$ OSTBC layers, where layer 1 is detected first, which allows us to eliminate the interference caused by the signal of layer 2. However, the proposed concept is applicable to arbitrary STCs and to an arbitrary number of layers $K$. For this reason, the decoder of layer 1 has to suppress the interference of layer 2 originally imposed on layer 1 and generate a signal that can be decoded using the STBC detector of [2]. This may be achieved by using the GSIC algorithm proposed in [10]. Then the decoder subtracts the remodulated contribution of the decoded symbols of layer 1 from the composite twin-layer received signal. Finally, the decoder applies direct STBC decoding to the second layer, since the interference imposed by the first layer has been eliminated. This group interference cancellation procedure can be generalized to arbitrary $N_{t}$ and $K$ values.

The proposed scheme is applicable to arbitrary STCs and an arbitrary number of layers $K$. For example, for $N_{t}=3$ transmit antennas, a twin-antenna-assisted STC can be employed in parallel with a single-antenna-aided transmission scheme. On the other hand, for a scheme employing $N_{t}=8$ transmit antennas, several configurations can be considered. One configuration can employ two four-antenna-aided STCs, while another configuration may employ four twin-antenna-aided STCs. The favored configuration will depend on the specific application as well as on the required performance and throughput.

The LSSTC scheme is characterized by a diversity gain, a multiplexing gain, and a beamforming gain. However, a drawback of the LSSTC design is the fact that the number of receive antennas $N_{r}$ should be at least equal to the number of transmit antennas $N_{t}$ for the interference canceller to work. This condition is not very practical for employing shirt-pocketsized mobile stations (MS) that are limited in size and complexity. The LSSTC scheme can be applied in a scenario where two base stations (BS) cooperate or a BS is communicating with a MIMO-aided laptop. Therefore, in order to allow communication between a BS and an MS accommodating fewer antennas than the transmitting BS while employing simple linear receivers, [11] presented a four-transmit tworeceive antenna aided scheme that combined the benefits of Alamouti's STBC [2] and V-BLAST [8]. The scheme of [11] was referred to as DSTTD and employed a simple linear decoder that used fewer antennas than the transmitter. The DSTTD receiver employed a two-stage decoding algorithm, where the first stage was interference cancellation, in order to cancel any interference imposed by each STBC layer on the other layer. The second decoding stage involved the maximum likelihood decoding of the STBC [2].

Furthermore, in order to allow multiple users to communicate employing a multifunctional MIMO, the layered steered space-time spreading (LSSTS) scheme described below can be employed. The LSSTS scheme combines the benefits of V-BLAST, STS, and beamforming with generalized multicarrier direct sequence CDMA (MC DS-CDMA) [15] for the sake of achieving a multiplexing gain, a spatial and frequency diversity gain, and a beamforming gain. The LSSTS design employs $N_{t}=4$ transmit antennas as well as $N_{r}=2$ receive antennas and a linear receiver to decode the received signal.

The system architecture of the LSSTS scheme can be seen in Fig. 2 with STS used as the component STC layers. The LSSTS scheme employs two twin-antenna-aided STS layers and $N_{r}=2$ receive antennas. The $L_{A A}$ numbers of elements of each AA are spaced at a distance of $\lambda / 2$ for the sake of achieving beamforming. The system can support $L$ users transmitting at the same time over the same carrier frequencies, because they can be differentiated by the user-specific spreading code $\overline{\mathbf{c}}_{l}$, where $l \in[1, L]$. Additionally, in the generalized MC DS-CDMA system con- 
sidered, the subcarrier frequencies are arranged to guarantee that the same STS signal is spread to and hence transmitted by the specific subcarriers having the maximum possible frequency separation so that they experience independent fading and achieve the maximum attainable frequency diversity.

The LSSTS system employs the generalized MC DS-CDMA scheme of [15], where the input data is serial-to-parallel converted to two parallel streams that are transmitted using twinantenna-aided STS [6]. The transmitted signal is spread to the two transmit antennas with the aid of the orthogonal spreading codes of $\left\{\overline{\mathbf{c}}_{l, 1} \overline{\mathbf{c}}_{l, 2}\right\}, l$ $=1,2, \ldots, L$. The spreading codes $\overline{\mathbf{c}}_{l, 1}$ and $\overline{\mathbf{c}}_{l, 2}$ are generated from the same user-specific spreading code $\overline{\mathbf{c}}_{l}$ as in [6].

The output of the two STS blocks modulate a group of subcarriers, where the subcarrier signals are superimposed on each other in order to form the complex-valued modulated signal for transmission. Finally, according to the $l$ th user's channel information, the signal of the $l$ th user is weighted by the transmit beamformer weight vector determined for each subcarrier of the $l$ th user, which is generated for the $n$th AA. Assuming that the system employs a modulation scheme transmitting $D$ b/symbol, the bandwidth efficiency of the LSSTS aided generalized MC DS-CDMA system is given by $2 D$ b/channel use.

Assuming that the $K$ users' data are transmitted synchronously over a dispersive Rayleigh fading channel, decoding is carried out in two steps. First, interference cancellation is performed according to [11], followed by the STS decoding procedure of [6]. Finally, after combining the $l=1$ st user's identical replicas of the same signal transmitted by spreading over the number of subcarriers, the decision variables corresponding to the symbols $\mathrm{PV}$ transmitted in each subblock can be expressed as $\widetilde{x}_{1}=\sum_{v=1}^{V} \widetilde{x}_{1, v}$, where $V$ is the number of subcarriers employed by the generalized MC DS-CDMA. Therefore, the decoded signal has a diversity order of $2 V$. More explicitly, second order spatial diversity is attained from the STS operation, and a diversity order of $V$ is achieved as a benefit of spreading by the generalized MC DS-CDMA scheme, where the subcarrier frequencies are arranged in such a way as to guarantee that the same STS signal is spread to and hence transmitted by the specific $V$ number of subcarriers having the maximum possible frequency separation, so they experience as independent fading as possible.

\section{RESULTS AND DISCUSSION}

In this section we compare the BER performance of the different MIMO schemes to that of the classic single-input single-output (SISO) system. We compare binary phase shift keying (BPSK) modulated systems, while considering transmissions over correlated Rayleigh fading channels associated with a normalized Doppler frequency of 0.01 .

According to Fig. 3, the V-BLAST system employing $\left(N_{t}, N_{r}\right)=(4,4)$ antennas has slightly better BER performance than the SISO system, despite its quadrupled throughput. Also observe in Fig. 3 that the slope of the BER curves of both the V-BLAST and SISO systems are simi-

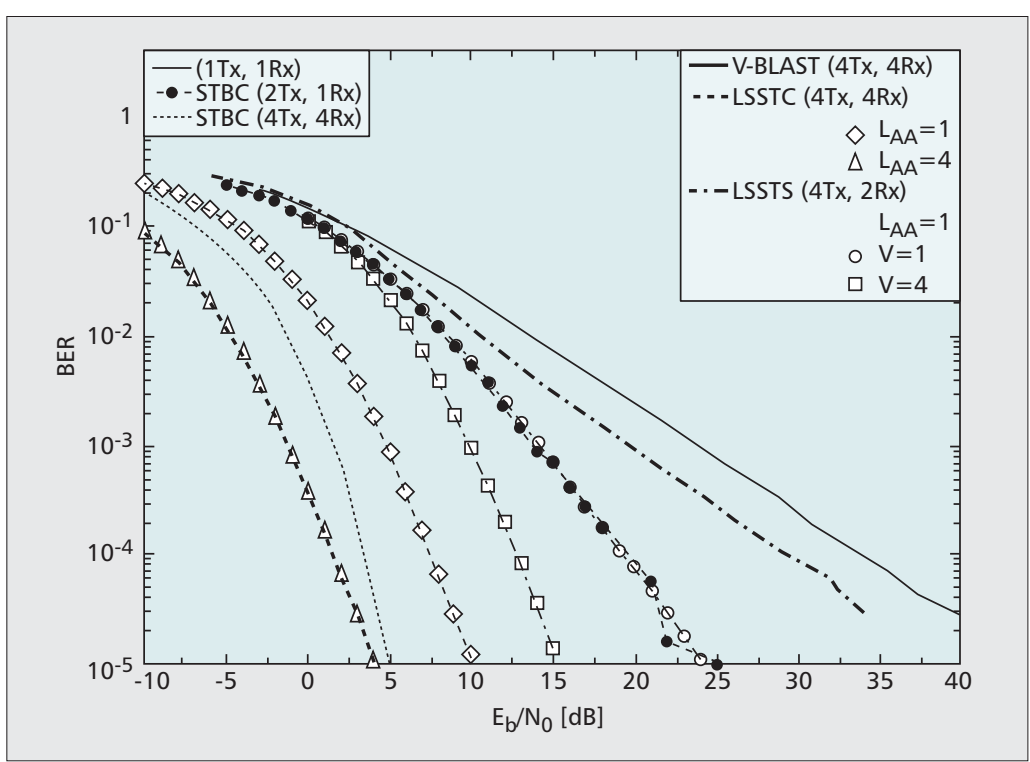

Figure 3. BER performance comparison of the SISO, STBC, V-BLAST, LSSTC, and LSSTS schemes, while communicating over a correlated Rayleigh fading channel associated with a normalized Doppler frequency of $\mathrm{f}_{\mathrm{d}}=0.01$.

lar, which suggests that V-BLAST does not attain a high diversity gain, but is capable of attaining a high multiplexing gain. Additionally, Fig. 3 shows that the STBC system employing $\left(N_{t}, N_{r}\right)=(2,1)$ attains better BER performance than the SISO and V-BLAST schemes due to the diversity gain attained by the STBC. Further diversity gain can be attained by the four-antenna-aided STBC employing four receive antennas, which results in a diversity order of 16. As shown in Fig. 3, the four-antenna-aided STBC scheme employing four receive antennas is capable of attaining around $15 \mathrm{~dB}$ gain at a BER of $10^{-5}$ over the twin-transmit-antenna-aided STBC system using $N_{r}=1$. However, a drawback of the four-antenna-aided system, while employing complex-valued constellations, is that it results in a throughput loss where four symbols are transmitted in eight time slots, resulting in a rate of $1 / 2$.

Observe in Fig. 3 that the LSSTS scheme employing $\left(N_{t}, N_{r}\right)=(4,2)$ and $V=1$ attains identical BER performance to that of the twintransmit-antenna-aided STBC system. This means that the LSSTS scheme employing $V=1$ has a diversity order of 2 similar to the twinantenna-aided STBC. On the other hand, the LSSTS scheme attains twice the throughput of the twin-transmit-antenna- aided STBC scheme. Additionally, when $V$ is increased from 1 to 4 , the achievable BER performance improves due to the additional frequency diversity gain attained.

A further performance improvement is attained by the LSSTC scheme in conjunction with $\left(N_{t}, N_{r}\right)=(4,4)$ compared to the LSSTS scheme. The LSSTC scheme employs more antennas than the LSSTS scheme and hence attains both a higher diversity order as well as better BER performance. Furthermore, Fig. 3 shows the performance improvements attained by beamforming, where the LSSTC scheme employing $L_{A A}=4$ attains around $6 \mathrm{~dB}$ perfor- 


\begin{tabular}{|c|c|c|c|c|c|c|c|c|c|}
\hline $\begin{array}{l}\text { The design of the } \\
\text { multi-functional }\end{array}$ & & $N_{t}$ & $N_{r}$ & $L_{A A}$ & $v$ & $\begin{array}{l}\text { Number } \\
\text { of layers }\end{array}$ & $\begin{array}{l}\text { Diversity } \\
\text { order }\end{array}$ & $\begin{array}{l}\text { Multiplexing } \\
\text { order }\end{array}$ & $\begin{array}{l}\text { Beamforming } \\
\text { gain }\end{array}$ \\
\hline MIMO scheme will & \multirow{3}{*}{ OSTBC } & 2 & $N_{r}$ & 1 & 1 & 1 & $2 \times N_{r}$ & 1 & 1 \\
\hline depend on the & & 4 & $N_{r}$ & 1 & 1 & 1 & $4 \times N_{r}$ & $1 / 2$ & 1 \\
\hline application & & 8 & $N_{r}$ & 1 & 1 & 1 & $8 \times N_{r}$ & $1 / 2$ & 1 \\
\hline the number of & \multirow{3}{*}{$\begin{array}{l}\text { V-BLAST } \\
\text { ZF-SIC }\end{array}$} & 2 & 2 & 1 & 1 & 2 & 1 & 2 & 1 \\
\hline antennas that can be & & 4 & 4 & 1 & 1 & 4 & 1 & 4 & 1 \\
\hline afforded on the & & 8 & 8 & 1 & 1 & 8 & 1 & 8 & 1 \\
\hline \multirow{4}{*}{$\begin{array}{l}\text { transmitter and } \\
\text { receiver. }\end{array}$} & \multirow{3}{*}{ LSSTC } & 4 & 4 & $L_{A A}$ & 1 & 2 & 4 & 2 & $L_{A A}$ \\
\hline & & 8 & 8 & $L_{A A}$ & 1 & 2 & 16 & 1 & $L_{A A}$ \\
\hline & & 8 & 8 & $L_{A A}$ & 1 & 4 & 4 & 4 & $L_{A A}$ \\
\hline & LSSTS & 4 & 2 & $L_{A A}$ & $v$ & 2 & $2 \times V$ & 2 & $L_{A A}$ \\
\hline
\end{tabular}

Table 1. Comparison of the gains achieved by various MIMO schemes.

mance improvement at a BER of $10^{-5}$ over its counterpart employing $L_{A A}=1$, provided that the direction of arrival (DOA) is perfectly known. Finally, a comparison between the STBC and LSSTC schemes using $\left(N_{t}, N_{r}\right)=(4,4)$ reveals that the STBC arrangement attains better performance than the LSSTC scheme employing $L_{A A}=1$. This is due to the fact that the STBC scheme has a higher diversity gain, while the LSSTC scheme attains a throughput four times that of its STBC counterpart.

Therefore, the design of the multifunctional MIMO scheme will depend on the application considered and the number of antennas that can be afforded on the transmitter and receiver. For example, as mentioned previously, for a handheld device the LSSTC scheme is not a practical choice due to the limited size and complexity of the small device. On the other hand, the LSSTS scheme can be considered a good choice for a system with a small-sized receiver that requires high robustness and throughput.

\section{DIVERSITY AND MULTIPLEXING OF MIMO SYSTEMS}

According to our previous discussions, different MIMO schemes have different structures and hence different BER as well as throughput performance. Explicitly, the OSTBC scheme is capable of attaining the highest possible spatial diversity gain while having no multiplexing gain; in fact, some STBC structures result in throughput loss. On the other hand, the V-BLAST scheme is capable of achieving the maximum possible multiplexing gain while attaining low diversity gain, depending on the choice of $\mathrm{V}$ BLAST decoder employed. Furthermore, several multifunctional MIMO schemes that can attain a combination of diversity, multiplexing, and beamforming gains have been introduced.
Table 1 compares the diversity, multiplexing, and beamforming gains of the different MIMO schemes for different configurations. In Table 1 $N_{t}$ and $N_{r}$ stand for the number of transmit and receive antennas, respectively, while $L_{A A}$ represents the number of elements per transmit AA and $V$ denotes the number of subcarriers employed by the generalized MC DS-CDMA system. Additionally, the number of layers represents the number of antenna layers used for transmitting different data symbols at the same time for the sake of attaining a multiplexing gain.

As shown in Table 1, the OSTBC schemes are capable of attaining a full diversity order of $\left(N_{t} \times N_{r}\right)$, while achieving no multiplexing or beamforming gain. In contrast, in the case of four- and eight-antenna-aided OSTBC schemes employing complex-valued constellations, the multiplexing gain is $1 / 2$, resulting in half the throughput of the SISO scheme. For example, in the four-antenna-aided OSTBC scheme, four symbols are transmitted in eight time slots; similarly for the eight-antenna aided STBC scheme, eight complex-valued symbols are transmitted in 16 time slots. On the other hand, as shown in Table 1, the V-BLAST scheme can attain a multiplexing gain of $N_{t}$, since the different antennas transmit different symbols in the same time slot. For example, for the V-BLAST scheme employing $\left(N_{t}, N_{r}\right)=(4,4)$, the transmitter transmits four different symbols from the four different antennas in the same time slot, which results in a quadrupled multiplexing gain in comparison to that of the SISO scheme. Observe in Table 1 that the diversity order of V-BLAST employing ZF-SIC is 1 for different $\left(N_{t}, N_{r}\right)$ configurations. The diversity order of the V-BLAST scheme employing ZF-SIC is $\left(N_{r}-N_{t}+1\right)$.

The LSSTC scheme combines the benefits of STBC, V-BLAST, and beamforming, as discussed earlier. This becomes clear in Table 1, where it is shown that the LSSTC scheme attains 
a diversity gain, a multiplexing gain, and a beamforming gain. In the case of the $\left(N_{t}, N_{r}\right)=(4,4)$ configuration, two twin-antenna STBC layers are implemented, which results in a diversity order of 4 and a multiplexing order of 2 . This is due to the fact that four symbols are transmitted from the four transmit antennas in two time slots. Additionally, when $L_{A A}$ elements are used per $\mathrm{AA}$, a beamforming gain can be attained. In the $\left(N_{t}, N_{r}\right)=(8,8)$ configuration, two different schemes can be implemented. The first scheme is a two-layer one with each layer consisting of a four-antenna STBC scheme. The other configuration employs four layers of the twin-antenna STBC scheme. The two configurations result in the different diversity and multiplexing gains shown in Table 1.

Finally, in the LSSTS scheme four transmit and two receive antennas are employed, where the transmit antennas are separated into two STS layers. The diversity order achieved by the LSSTS scheme is $(2 \times V)$ as discussed earlier. The multiplexing order of the LSSTS scheme is 2 , since four symbols are transmitted in two time slots. Moreover, the LSSTS scheme is capable of attaining a beamforming gain when $L_{A A}>1$ elements per AA are used.

\section{CONCLUSION}

In this article a brief classification of the family of MIMO schemes is presented based on their attainable diversity, multiplexing, and beamforming gains. We also investigate the design of the novel class of multifunctional MIMO schemes that are capable of combining the benefits of several MIMO schemes and hence attaining diversity, multiplexing, and beamforming gains. More explicitly, we introduce the dual-functional MIMO scheme of [10] followed by the LSSTC scheme that combines the benefits of STBC, VBLAST, and beamforming. Then we discuss the design of the DSTTD followed by the LSSTS arrangement that combines the advantages of STS, V-BLAST, and beamforming with those of generalized MC DS-CDMA while supporting multiple users. Finally, a comparison between the BER performance as well as the diversity, multiplexing, and beamforming gains of the different MIMO schemes reveals that multifunctional MIMOs are capable of attaining improved performance over the now classic standalone STBC and V-BLAST schemes.

\section{REFERENCES}

[1] L. Hanzo, T. H. Liew, and B. L. Yeap, Turbo Coding, Turbo Equalisation and Space Time Coding for Transmission over Fading Channels, Wiley-IEEE Press, 2002.

[2] S. M. Alamouti, "A Simple Transmit Diversity Technique for Wireless Communications," IEEE JSAC, vol. 16, no. 8, 1998, pp. 1451-58.

[3] V. Tarokh, H. Jafarkhani, and A. R. Calderbank, "Spacetime Block Codes from Orthogonal Designs," IEEE Trans. Info. Theory, vol. 45, no. 5, 1999, pp. 1456-67.

[4] V. Tarokh, N. Seshadri, and A. R. Calderbank, "Space-Time Codes for High Data Rate Wireless Communication: Performance Criterion and Code Construction," IEEE Trans. Info. Theory, vol. 44, Mar. 1998, pp. 744-65.
[5] B. Hassibi and B. M. Hochwald, "High-Rate Codes that Are Linear in Space and Time," IEEE Trans. Info. Theory, vol. 48, July 2002, pp. 1804-24.

[6] B. Hochwald, T. L. Marzetta, and C. B. Papadias, "A Transmitter Diversity Scheme for Wideband CDMA Systems Based on Space-Time Spreading," IEEE JSAC, vol. 19, no. 1, 2001, pp. 48-60.

[7] H. Jafarkhani, "A Quasi-Orthogonal Space-Time Block Code," IEEE Trans. Commun., vol. 49, no. 1, 2001, pp. 1-4.

[8] P.W. Wolniansky et al., "V-BLAST: An Architecture for Realizing Very High Data Rates over the Rich-Scattering Wireless Channel," Int'l. Symp. Signals, Sys., Electronics, Pisa, Italy, Sept. 1998, pp. 295-300.

[9] L. Hanzo, J. Blogh, and S. Ni, 3G, HSPA and FDD versus TDD Networking: Smart Antennas and Adaptive Modulation, Wiley-IEEE Press, 2008.

[10] V. Tarokh et al., "Combined Array Processing and Space-Time Coding," IEEE Trans. Info. Theory, vol. 45, no. 4, 1999, pp. 1121-28.

[11] E. N. Onggosanusi, A. G. Dabak, and T. A. Schmidl, "High Rate Space-Time Block Coded Scheme: Performance and Improvement in Correlated Fading Channels," IEEE WCNC, vol. 1, Mar. 2002, pp. 194-99.

[12] G. Jongren, M. Skoglund, and B. Ottersten, "Combining Beamforming and Orthogonal Space-Time Block Coding," IEEE Trans. Info. Theory, vol. 48, Mar. 2002, pp. 611-27.

[13] J. Liu and E. Gunawan, "Combining Ideal Beamforming and Alamouti Space-Time Block Codes," Elect. Lett., vol. 39, Aug. 2003, pp. 1258-59.

[14] L. Hanzo et al., Near-Capacity Multi-Functional MIMO Systems: Sphere-Packing, Iterative Detection and Cooperation, Wiley-IEEE Press, 2009.

[15] L.-L. Yang and L. Hanzo, "Performance of Generalized Multicarrier DS-CDMA over Nakagami-m Fading Channels," IEEE Trans. Commun., vol. 50, June 2002, pp. 956-66.

\section{BIOGRAPHIES}

MOHAMMED EL-HAJJAR received his B.Eng. degree (with distinction) in electrical engineering from the American University of Beirut (AUB), Lebanon, and an M.Sc. degree (with distinction) in radio frequency communication systems from the University of Southampton, United Kingdom. Since October 2005 he has been working toward his Ph.D. degree with the Communications Group, School of Electronics and Computer Science, University of Southampton. He is the recipient of several academic awards from AUB as well as the University of Southampton. His research interests include sphere packing modulation, space-time coding, differential space-time spreading, adaptive transceiver design, and cooperative communications. In 2008 he completed his Ph.D. thesis and joined Ensigma in Chepstow, Wales, United Kingdom as a wireless system architect.

LAJOS HANZO [F] (Ih@ecs.soton.ac.uk), FREng, FIET, received his degree in electronics in 1976, his doctorate in 1983, and his D.Sc. degree in 2004. During his 34-year career in telecommunications he has held various research and academic posts in Hungary, Germany, and the United Kingdom. Since 1986 he has been with the School of Electronics and Computer Science, University of Southampton, where he holds the chair in telecommunications. He has coauthored 19 Wiley-IEEE Press books on mobile radio communications totaling in excess of 10,000 pages, published 684 research papers at IEEE Xplore, acted as TPC Chair of IEEE conferences, presented keynote lectures, and been awarded a number of distinctions. Currently he is directing an academic research team working on a range of research projects in the field of wireless multimedia communications sponsored by industry, the Engineering and Physical Sciences Research Council (EPSRC) United Kingdom, the European IST Program, and the Mobile Virtual Centre of Excellence (VCE), United Kgindom. He is an enthusiastic supporter of industrial and academic liaison, and he offers a range of industrial courses. He is also an IEEE Distinguished Lecturer as well as a Governor of both the IEEE Communications Society and the VTS. He is the acting Editor-in-Chief of IEEE Press. For further information on research in progress and associated publications please refer to http://www-mobile.ecs.soton.ac.uk.
A comparison

between the BER

performance as well

as the diversity,

multiplexing, and

beamforming gains

of the different

MIMO schemes

reveals that multi-

functional MIMOs

are capable of

attaining an improved

performance over

the now classic

stand-alone STBC

and V-BLAST

schemes. 